\title{
The low impact of the guidelines: does it concern only colorectal polypectomy?
}

\section{(ㄷ)(1) $\odot$}

\author{
Authors \\ Cesare Hassan ${ }^{1}$, Raf Bisschops ${ }^{2}$ \\ Institutions \\ 1 Endoscopy Unit, Nuovo Regina Margherita Hospital, \\ Rome, Italy \\ 2 Department of Gastroenterology, University Hospitals \\ Leuven, Leuven, Belgium \\ Bibliography \\ DOI https://doi.org/10.1055/a-1096-0706 | \\ Endoscopy International Open 2020; 08: E456-E457
}

\author{
(c) Georg Thieme Verlag KG Stuttgart · New York \\ elSSN 2196-9736 \\ Corresponding author \\ Cesare Hassan, ONRM Hospital - Gastro, Via Morosini 30, \\ Rome 00153, Italy \\ Fax: +390658446533 \\ cesareh@hotmail.com
}

Every year, approximately 4 million Europeans people undergo an immunochemical fecal test (FIT) within a population-based program, and we can estimate that $5 \%$ to $7 \%$ will end up with a FIT + colonoscopy [1]. Of note, due to the enriched-disease effect of FIT, $20 \%$ to $30 \%$ of these colonoscopies will present the endoscopist with an advanced adenoma that requires complete and safe endoscopic resection. Do we have an adequate standard of endoscopic resection to serve our target population that we proactively invite?

There is little - if any - doubt that dissemination of recommendations and education from scientific societies to the medical community led to a massive improvement of the clinical and technical standards of colonoscopy. Less than two decades ago, we were struggling in reaching the cecum as the only primary goal of our diagnostic colonoscopy and today, concepts like detection of at least one adenoma or post-resection recurrence rates dominate the field of screening colonoscopy [2]. Split preparation, wide-angle high-definition scopes, prolonged withdrawal time, recognition of non-polypoid lesions, characterization of the surface patterns of the lesions, coupled with an impressive variety of devices and techniques for endoscopic resection upgraded the standard of screening colonoscopy to an unprecedented level.

We may reasonably agree that the current standard of screening colonoscopy has become acceptable, and evidence of substantial reduction of colorectal cancer (CRC) incidence and mortality would support it. However, most of us would similarly argue that what is acceptable is not necessarily desirable or optimal! Although at first glance, the difference between an acceptable and desirable level may appear negligible, it actually results in big differences in terms of clinical outcome.
The simplest example is represented by the profound reduction of post-colonoscopy interval CRC achieved by the very high - as compared with average - detector as measured by individual adenoma detection rate. Isn't such a difference between what is acceptable and what is optimal the main irrational driver pushing most patients to those few endoscopists regarded as "experts"?

It is time to be more ambitious! The next challenge for colonoscopy is to shift what is perceived as an acceptable to a desirable standard. It would be naïve to expect that this can be achieved by mere production of one or more documents, whereas it may require a much deeper penetration in the fiber of the endoscopy community, irrespective of its willingness.

A good example of where we stand is provided by the international survey on the attitude of endoscopists by over 700 endoscopists in seven countries reported in this issue of the Journal [3]. When considering the recent ESGE guidelines on polypectomy and endoscopic mucosal resection (EMR) as a template for an optimal standard [4], the authors inferred the technical and cultural approach, with quite controversial and somewhat disappointing results [3].

The evidence that less than $50 \%$ of resections of small to diminutive polyps are appropriately performed by the recommended cold-snaring deserves some comments [3]. None of us would consider it as clinically relevant due to the remote, if any, chance that an incomplete resection of these tiny lesions would cause an interval CRC. On the other hand, such technical mismanagement is a proxy for what a step-up approach can eventually achieve. Here as step-up approach we define the process initiated by dissemination of a guideline followed by educational interventions by international, national, and local 
societies, including evidence-based courses, e-learning, translational and other activities. According to the published survey [3], such a step-up approach succeeded in persuading approximately $40 \%$ of the endoscopists, suggesting that there are some major barriers for the remaining majority. Of course, we can see the glass half full, as usually a step-up approach is not so much demanding in terms of required resources, acting more as a tool than a formal strategy. But the glass should also be seen as half empty! The fact that the majority of the endoscopists, more or less consciously, are not adherent to such a technically simple and convenient recommendation - coldsnaring rather than forceps-biopsy - indicates the possible coalescence of strong personal and cultural barriers. What if the majority of the endoscopists do not consider as a mindful standard the one that is based on evidence-based data?

The catastrophic consequences of the half-empty glass can be immediately recognized when shifting our attention from the small to the largest and more complex polyps. Before looking at the survey data, let us just briefly return to the European organized FIT-based programs. Comparative analysis of different centers showed unexpected variability in the rate of postcolonoscopy surgeries, as well as in the rate of major adverse events. Should we assume that some endoscopists are referring patients with early colorectal neoplasia to useless surgeries? Is there variability among centers in safety of complex resections? According to the survey, these risks exist and affect a not negligible proportion of our community [3]. As shown by the authors, overuse or under-use of endoscopic resection for complex polyps depends not only on the same personal disbeliefs against evidence-based standard, but also on suboptimal competence in differentiating between early and deeply invasive CRC. It is unlikely that lack of cognitive competence, such as that required for characterization of colorectal neoplasia, can be improved by disseminating further guidelines or educational courses. It is affected by multidimensional factors, such as volume of complex resections, setting of training and retraining, auditing of efficacy and safety outcomes, and skills of medical and non-medical personnel.

A much stronger response is needed! Can we replace the traditional step-up approach with a top-down approach to protect our patients from potentially catastrophic mismanagement? Appropriateness of endoscopic strategies that heavily affect clinical outcomes cannot be dependent upon goodwill of the endoscopist. Some formal strategies must be adopted to en- sure optimal management. Safety, first! The simplest example is to include in accreditation of screening centers the requirement for patients with complex polypoid or non-polypoid lesions to be discussed in a multidisciplinary setting. The second is to define a minimal cut-off of volume for endoscopic or surgical resections. The third is to formally require that screening centers adopt quality indicators specifically dedicated to management of complex lesions, that is surgery referral of T0(in situ) CRC, post-EMR recurrence rate, and major adverse events.

\section{Conclusion}

In conclusion, it would be too simplistic to underrate technical pitfalls in endoscopic resection standards as a clinically marginal detail of screening colonoscopy. To the contrary, such pitfalls are strong predictors of suboptimal cognitive attitudes that may compromise the core of CRC screening efficacy, which is complete and conservative removal of the advanced neoplasia. It should be the mind not the hand that dictates an effective and safe resection!

\section{Competing interests}

The authors declare that they have no conflict of interest.

\section{References}

[1] Senore C, Basu P, Anttila A et al. Performance of colorectal cancer screening in the European Union Member States: data from the second European screening report. Gut 2019; 68: 1232-1244

[2] Kaminski MF, Thomas-Gibson S, Bugajski M et al. Performance measures for lower gastrointestinal endoscopy: a European Society of Gastrointestinal Endoscopy (ESGE) quality improvement initiative. United European Gastroenterol ] 2017; 5: 309-334

[3] Tate DJ, Desomer L, Heitman SJ et al. Clinical implications of decision making in colorectal polypectomy: an international survey of Western endoscopists suggests priorities for chang. Endosc Int Open 2020; 8: E445-E455

[4] Ferlitsch M, Moss A, Hassan C et al. Colorectal polypectomy and endoscopic mucosal resection (EMR): European Society of Gastrointestinal Endoscopy (ESGE) Clinical Guideline. Endoscopy 2017; 49: 270-297 